Cahiers de recherches médiévales

\title{
La famille et le village
}

Identification et dénomination de l'individu dans la Terre de l'abbaye de Saint-Claude (XIII ${ }^{\mathrm{e}} \mathrm{XVI}{ }^{\mathrm{e}}$ siècles)

\section{Vincent Corriol}

\section{(2) OpenEdition}

1 Journals

Édition électronique

URL : https://journals.openedition.org/crm/1553

DOI : $10.4000 / \mathrm{crm} .1553$

ISSN : 1955-2424

Éditeur

Honoré Champion

Édition imprimée

Date de publication : 15 mars 2003

Pagination : 53-69

ISSN : $1272-9752$

\section{Référence électronique}

Vincent Corriol, «La famille et le village », Cahiers de recherches médiévales [En ligne], 10 | 2003, mis en ligne le 08 octobre 2007, consulté le 15 décembre 2022. URL : http://journals.openedition.org/crm/ 1553 ; DOI : https://doi.org/10.4000/crm.1553

Ce document a été généré automatiquement le 15 décembre 2022.

Tous droits réservés 


\title{
La famille et le village
}

\author{
Identification et dénomination de l'individu dans la Terre de l'abbaye de \\ Saint-Claude (XIII ${ }^{\mathrm{e}}$ XVI ${ }^{\mathrm{e}}$ siècles)
}

\section{Vincent Corriol}

1 C'est au cours des $\mathrm{XII}^{\mathrm{e}}$ et $\mathrm{XIII}{ }^{\mathrm{e}}$ siècles que s'affirme pour plus de trois siècles l'indépendance de la Terre de Saint-Claude ${ }^{1}$ l'abbé impose son pouvoir temporel au sein d'un vaste espace encore en grande partie désert et s'affranchit de toute tutelle autre que celle de l'empereur. La Terre de Saint-Claude constitue alors un vaste ensemble d'environ $1600 \mathrm{~km}^{2}$ d'un seul tenant dans la montagne jurassienne, où s'exerce sans partage l'autorité du monastère, autour de la figure du seigneur-abbé. Cette construction d'une principauté territoriale homogène, où l'abbé règne en prince souverain, s'accompagne d'un renforcement du pouvoir seigneurial sur les hommes, nobles ou paysans, tous soumis à la tutelle seigneuriale à travers les liens de la vassalité ou ceux du servage.

2 La riche documentation laissée par l'administration monastique offre un aperçu intéressant de la vie rurale des campagnes de la montagne jurassienne au moment même de leur colonisation'. Un aspect frappant de cette vie rurale, tel qu'il ressort de cette documentation, est la très faible présence de structures communautaires autonomes face au pouvoir abbatial. L'absence de mouvements de révolte face au pouvoir seigneurial qui maintient un servage général et toujours vivant, le très faible nombre de communautés ou d'individus affranchis avant le début du XVI siècle, sont autant d'aspects de cet effacement des structures communautaires. La tutelle seigneuriale est-elle si forte qu'elle a empêché le développement d'une autonomie paysanne? Pourtant de loin en loin se rencontrent de rares mentions de syndics, de procureurs, ou de bois ou pâtures communales, preuve d'une gestion par la communauté villageoise de ressources propres. Absente des textes, la communauté est donc une réalité vivante qui fonctionne au quotidien, ordonnant un cadre de vie qui constitue le premier rouage du système politique et administratif entourant l'individu.

3 Toutefois la communauté municipale, exerçant un pouvoir de gestion et de décision, se distingue nettement de la communauté vécue, ressentie et exprimée par les acteurs de la vie rurale. Plus que par l'exercice d'un pouvoir, réduit par les prérogatives étendues 
du pouvoir abbatial, c'est autour de la structuration de l'espace vécu que s'organise la communauté. La notion de territoire est au cœur de cette structuration de l'espace et de l'organisation communautaire. C'est par cette notion de territoire que se positionne l'individu, dans un réseau à plusieurs niveaux. La famille, le village, la seigneurie composent les trois étages d'un réseau communautaire tel que le conçoivent et le dessinent les paysans jurassiens.

La faiblesse apparente des structures communautaires

4 Le pouvoir seigneurial des abbés de Saint-Claude ne cesse de se renforcer sur l'espace constitué par la seigneurie entre la fin du XII siècle et le début du XIV siècle. Un des aspects les plus marquants de ce pouvoir est la constitution d'un système social contraignant ordonné autour de la figure du seigneur-abbé. Le renforcement des liens d'homme à homme, via le système vassalique, et la généralisation du servage sur les paysans et tenanciers de la terre abbatiale, conduisent à médiatiser tout rapport social autour d'un point nodal constitué par le monastère, et symbolisé par l'abbé.

5 Cette mise en place semble pourtant se faire sans heurts. Aucune trace d'un quelconque mouvement de contestation du pouvoir seigneurial ne peut être relevée. Le très faible nombre de communautés affranchies marque bien la très grande méfiance du pouvoir seigneurial face à une quelconque forme d'autonomie des communautés d'habitants. Les seules localités affranchies sont des cas particuliers. Les deux bourgs de SaintClaude, siège du monastère, affranchi au plus tard en $1310^{3}$, et de Moirans, siège du principal château et résidence de l'abbé, affranchi en $1351^{4}$, sont les seuls exemples de concession de franchises par l'abbé, sans que l'on puisse réellement saisir l'ampleur de la pression communautaire. On ne sait rien des conditions qui ont présidé à la concession de cette autonomie. Les deux chartes se réfèrent à des documents antérieurs dont il ne subsiste aucune trace.

6 L'abbé n'entend pas partager son pouvoir. En dehors des deux villes déjà citées, un seul village $^{5}$, en 1393 seulement $^{6}$, se voit confier officiellement une relative autonomie administrative strictement encadrée. En autorisant les habitants de Longchaumois et Orsières à élire de trois ans en trois ans quatre «honnêtes hommes " pour régler les affaires de ces deux villages ${ }^{7}$, c'est l'autonomie d'une communauté parmi les plus importantes de sa seigneurie que l'abbé reconnaît ici, sans que cela s'accompagne d'ailleurs d'une quelconque liberté. L'emploi du terme communitas est suffisamment rare dans la documentation san-claudienne pour qu'il soit noté ici'; on lui préfère en général le terme de village (villa en latin).

7 Il n'y a guère que sous la pression que l'abbé accepte de concéder plus largement des franchises. Mais cette pression n'est pas exercée par les paysans: elle provient des princes voisins, en premier lieu de la famille comtale de Chalon, possessionnée au nord de la Terre de Saint-Claude et concurrente dans la conquête des terres vierges des montagnes jurassiennes. Les villages de Mouthe, Châtelblanc ou Rochejean, que l'abbé affranchit progressivement, entre 1296 et $1364^{10}$, sont des exemples de cette frilosité abbatiale. Excentrés en limite nord de la Terre de Saint-Claude, éloignés du monastère, ces villages co-détenus par les comtes de Chalon depuis 1237 et $1266^{11}$ ont été affranchis depuis plusieurs années par celui-ci. Dans ces zones, le pouvoir de l'abbé est déjà marginal, concurrencé par un prince trop puissant pour qu'il puisse lutter. L'abbé en confirmant l'affranchissement ne fait que reconnaître un état de fait, marquant la perte d'influence du monastère dans cette zone. 
Les servitudes communautaires

Ces cas particuliers ne font que révéler la méfiance seigneuriale face à une quelconque forme d'organisation communautaire dans les villages de la Terre de Saint-Claude. L'impression de faiblesse des structures communautaires est renforcée par la rareté des droits et servitudes communautaires clairement lisibles. La seule mention de mise en défens de communaux se trouve dans la charte du bourg abbatial de Saint-Claude, cas unique dans les archives ${ }^{12}$. Il faut attendre la fin du XIVe siècle pour trouver les premières mentions indiscutables d'une quelconque propriété commune des communautés paysannes ${ }^{13}$. Ces mentions restent cependant très rares, même au début $\mathrm{du} \mathrm{XVI} \mathrm{X}^{\mathrm{e}}$ iècle $^{14}$. Est-ce un signe de la rareté de ces terres communes ou est-ce simplement un effet de source? La gestion de l'inculte par les communautés paysannes est une source de renseignements sur le degré d'autonomie de ces communautés rurales.

9 Les servitudes communautaires sont peu nombreuses : le droit de vaine pâture existe, il fait l'objet d'un litige en 1414 entre les habitants du village de Lavans et les officiers de l'abbé ${ }^{15}$, mais c'est le seul exemple dans toute la documentation san-claudienne. En présence de vastes immensités forestières, et de prairies nombreuses, on conçoit aisément qu'il ne soit pas vraiment une source de conflit ou de préoccupation. Il en va de même pour les droits d'usages dans les forêts, qui toutes appartiennent à la directe seigneuriale de l'abbé. Il est rare que ceux-ci fassent l'objet de règlements particuliers : les forêts mises en défens par l'abbé sont rares, ce qui n'est pas étonnant dans le contexte jurassien de défrichement forestier ${ }^{16}$. Les bois délimités ou concédés sont toujours des bois seigneuriaux, dont l'abbé laisse une partie à la jouissance des paysans. Cette propriété seigneuriale des bois apparaît dès le début du XIV ${ }^{\mathrm{e}}$ siècle. En 1303, un accord est conclu avec la chartreuse de Vaucluse, voisine de la terre de Saint-Claude. De manière à respecter le désert cartusien, le bois de Bonnans, qui sépare la chartreuse des terres san-claudienne, est mis en défens. La même année, un accord sur la justice avec des vassaux de la terre de Saint-Claude, précise les bois laissés à disposition de ces vassaux et de leurs hommes, laissant de côté ce bois de Bonnans.

10 Tous ces accords ne se font qu'entre seigneurs. Il faut attendre la seconde moitié du $\mathrm{XIV}^{\mathrm{e}}$ siècle pour que les communautés se voient confier de manière formelle, fixe et définitive, des droits d'usages spécifiques, dans des bois dits communaux. Le premier exemple date de 1365 : les habitants de Coiron et Meussia se plaignent des dégâts commis par les bêtes sauvages dans leurs champs, et de l'envahissement des bois sur les terres cultivées. L'abbé accède à leur demande et les autorise à " tranchier, ésaigier, ardoyer et fayre toute leur bonne volentez de tous boys tant chasne comme aultres qui sont es fins desdictes villes ou environ, escepté bois de scirée, de pas ou de passages ». L'abbé leur accorde aussi le droit de «tranchier en leurs prels les branches des chasnes sy haut comm'ilz pourront tranchier dois terre sans monter sur le arbre et fayre leur volonté des dictes branches tranchiées" $"$. En 1388, à la suite d'un conflit entre les communautés de différents villages d'une part, et les officiers seigneuriaux de l'abbé de l'autre, des droits similaires sont reconnus dans le même secteur. Les droits d'usages des habitants de ces villages sont rappelés et spécifiés, après définition des limites des bois bannaux et des bois communaux :

in omnibus nostris nemoribus communibus parrochie predicte superius limitatis dicti homines nostri villarum predictarum omnia nemora et omnes arbores quacumque scindere et capere possint et facere folis seu exartagia et facere de nemore planum pro ut sibi ipsis et 
eorum heredibus placuerit eorum propria auctoritate et absque aliqua offensa committenda, et branchias de quercu in dictis eorum pratis existentis in nemoribus nostris bannalibus scindere pro ut consueverunt ${ }^{18}$.

11 Les rares mentions datent donc de la seconde moitié du XIV ${ }^{\mathrm{e}}$ siècle, et concernent toute la châtellenie de Moirans, partie la plus favorisée de la Terre de Saint-Claude. Localisée dans la partie sud-ouest de la Terre, c'est la zone la plus basse en altitude, la plus chaude et la moins humide. C'est aussi la plus anciennement colonisée, dès le haut Moyen Âge, et la plus densément peuplée, ce qui explique sans doute ces règlements. Il est à noter cependant que ces règlements ne se font pas à la suite de conflits violents entre communautés, mais bien entre l'abbé d'une part, et les communautés de l'autre. Il semble donc que même dans la zone où les bois sont vraisemblablement les moins étendus, les communautés ne sont pas en rivalité dans la gestion de l'inculte. Les restrictions sur les bois ne sont d'ailleurs pas très importantes. Hors de cette zone précise, les droits des communautés dans les bois ne sont jamais détaillés, ni même mentionnés. La très grande abondance de la forêt rend sans doute inutile toute mise en défens.

Les possessions communes concernent avant tout des pâturages, et les droits de pâtures qui en découlent. La première mention vient d'un conflit entre les habitants des Bouchoux et ceux des villages voisins, Choux et Coirières. En 1391, ce conflit est porté devant la justice abbatiale, pour trancher le différend. Les habitants des Bouchoux se plaignent que leurs voisins ne leur laissent plus l'accès à leurs pâtures communes, situées sur les territoires de ces villages, et leur coupent l'accès aux chemins publics, les privant de la libre circulation des marchandises nécessaire à leur activité $^{19}$. C'est par ailleurs le seul exemple de conflit de pâtures entre deux communautés san-claudiennes avant le milieu du siècle suivant. En 1453, les habitants des communautés de Longchaumois et Orsières d'une part, et ceux de Septmoncel de l'autre, se disputent la possession du lieu-dit « en Belbochet », situé en limite de deux communautés. Elles décident de porter leur différent devant le cellérier du monastère, ceux de Longchaumois affirmant que dictum locum de Belbochet est de comminibus ipsorum de Longocamelo et qu'il fait partie de leur pasqueragia. Ils accusent deux particuliers de Septmoncel de s'être approprié le lieu-dit en question. La possession du pré est finalement reconnue à Longchaumois, et les limites entre les deux communautés sont précisées.

13 À l'exception de ces deux exemples, la possession des communaux n'est pourtant pas source de conflit. L'abondance de la terre disponible y est sans doute pour beaucoup, et la pression démographique n'est pas telle que les communautés doivent se disputer l'espace $^{20}$. Il est d'ailleurs significatif que les mentions de communaux ne viennent pas tant des conflits, mais de la concession de terres seigneuriales à la libre disposition des communautés : c'est le cas en 1412 et en 1414 à Saint-Lupicin et à Lavans ${ }^{21}$, dans des zones pourtant peu élevées en altitude et anciennement mise en valeur.

Se défendre et se faire représenter

14 Cela ne signifie pourtant pas que les communautés d'habitants sont inexistantes, et que les individus ne se réfèrent pas à des structures plus larges pour se situer dans l'espace géographique et social. Les communautés d'habitants n'hésitent pas à se faire représenter en cas de besoin: en témoignent les rares mentions de syndics, prud'hommes ou échevins, qui apparaissent dans la seconde moitié du XIV ${ }^{\mathrm{e}}$ siècle, à l'occasion de conflits opposant un groupe d'habitants à un autre, ou lors d'une 
transaction avec le seigneur. La première mention date de 1347 : trois représentants du village de Lavancia sont désignés pour régler le conflit qui les oppose au village voisin de Dortans; ils agissent nomine totuis communitatis ville de Lavancia ${ }^{22}$. Cette désignation de représentants pour régler un problème ponctuel est toujours en vigueur en 1364 : dix procuratores electi et probati nominati per universitas dicte parrochie sont désignés pour régler les questions liées aux moulins de Septmoncel par les villages de Septmoncel et Montépile ${ }^{23}$.

Rapidement pourtant, il semble que la communauté désigne des représentants permanents: c'est ce que l'on peut conclure des mentions de "gentilshommes et prodomes " ${ }^{24}$, $d^{\prime}$ "échevins " ${ }^{25}$, de "prud'hommes et échevins " ${ }^{26}$, "prud'hommes, échevins et conseillers ${ }^{27}$ que l'on rencontre parfois. Le cas de Longchaumois est le mieux connu: en 1390, l'abbé concède aux habitants de Longchaumois et d'Orsières le droit d'élire quatre représentants, que l'on voit à l'œuvre dès 1393 dans un conflit au sujet de la possession d'un pré avec l'infirmier de l'abbaye, puis en 1453 face à Septmoncel. Ces mentions deviennent plus fréquentes à la fin du XV siècle, comme si l'institution de représentants permanents devenait plus fréquente. Toutefois on parle encore souvent des "habitants de tels villages", des "hommes et habitants de telle ville», sans plus de précision. La représentation de la communauté n'est pas systématique: est-ce une représentation ponctuelle, ou le mode normal de fonctionnement de la communauté ? Les représentants ont-ils été élus spécialement pour trancher l'affaire en question, ou sont-ils les représentants légaux et permanents de la communauté, qui agissent ici dans le cadre de leurs attributions? Difficile de trancher.

16 La communauté est donc susceptible de s'organiser ; mais les témoignages ne sont ni très nombreux, ni très explicites. En l'absence de ces structures communautaires fortes, et surtout de servitudes communautaires, il est difficile de cerner ce qui fédère la communauté d'habitants, qui n'hésite pourtant pas à se faire représenter à l'extérieur, preuve de la conscience de sa singularité.

La notion de territoire

17 Une notion qui ressort incidemment dans divers documents est pourtant capitale en ce domaine ; c'est celle de finage et de territoire. Les deux termes apparaissent souvent en binôme dans les documents médiévaux, sous la forme finagium et terriorium ${ }^{28}$, finagium seu territorium ${ }^{29}$, en complément l'un de l'autre; ils servent couramment à définir le cadre d'habitation. Cette formulation apparaît dès le milieu du XIII ${ }^{e}$ siècle, et reste vivante jusqu'à la fin du XV $\mathrm{XV}^{\mathrm{e}}$ siècle au moins. Dès le XIII ${ }^{\mathrm{e}}$ siècle, territorium, tout comme finagium, est employé en complément de villa, sous la forme territorium villae. Le territoire semble alors être un synonyme du finage villageois. Mais ces deux termes sont rarement employés de manière indépendante; dès la seconde moitié du XIII ${ }^{e}$ siècle, l'expression territorium seu finagium devient très courante, et reste employée jusqu'au début du XVI ${ }^{\mathrm{e}}$ siècle.

18 Malgré l'équivalence introduite par seu, les deux termes semblent au contraire se renforcer, désignant deux réalités légèrement distinctes. Ainsi, si le territoire est une notion qui tend à s'imposer, il n'en va pas de même pour le finage. Passé le XIII ${ }^{\mathrm{e}}$ siècle, le terme de finage est accompagné de celui de territoire ${ }^{30}$. Cette dernière notion s'impose pour désigner l'espace dominé par le village, sous la forme finagium seu territorium villae; mais le terme de territoire est aussi employé seul. Plus que la 
communauté (le terme communitas est très peu fréquent ${ }^{31}$, de même que celui d'universitas, que l'on rencontre à une seule reprise ${ }^{32}$ ), c'est cette notion de territoire qui est au cœur de la définition du village. Ce terme de territorium est le plus usuel pour localiser une terre à l'intérieur de la seigneurie san-claudienne, sous la forme in territorio dictae villae. C'est la formule systématiquement en usage dans les transactions de la terre pour situer les biens et parcelles échangés : c'est par le territoire du village que l'on se repère dans l'espace.

19 Le territoire désigne le village dans toute son extension, espace spécifique, unité géographique et sociale particulière. Cette dimension est soulignée par la formule villa et territorio ${ }^{33}$, qui désigne l'ensemble du ressort d'un village. Plus qu'une simple précision géographique, le terme recouvre donc une réalité plus complexe ; ainsi, par trois fois, le terme de territoire est synonyme de juridiction. En 1242, le comte de Bourgogne Jean de Chalon reconnaît ne posséder aucun droit dans la châtellenie de Moirans, in dominio seu territorio de Morenc ${ }^{34}$. En 1334, les terres relevant du diocèse de Genève, dont la limite est la frontière orientale de la Terre de Saint-Claude, sont dites situées dans le territoire du diocèse ${ }^{35}$. En 1475 enfin, l'équivalence entre les deux termes est explicite : lors d'un procès de mainmorte opposant le couvent de Saint-Claude et un particulier de Genève, le ressort de la seigneurie de l'abbé, c'est-à-dire l'ensemble de la terre de Saint-Claude, est désigné par le terme de territoire, sous la forme territorium sive juridictionem terre Sancti Eugendi Jurensis ${ }^{36}$. Appliqué au village, ce même terme de territoire désigne donc non seulement le finage, mais toute l'aire géographique à l'intérieur de laquelle le village entend s'imposer.

Le territoire est une notion difficile à caractériser ${ }^{37}$. Il désigne l'ensemble des terres d'un village, y compris les bois et les terres incultes, sur lesquelles les habitants de ce village entendent garder sinon une exclusivité, du moins une priorité. Ces terres appartiennent au village, elles lui sont attachées. Finalement, ce qui caractérise le territoire, c'est la frontière : il y a une limite du territoire, au-delà de laquelle on sort $\mathrm{du}$ territoire du village pour entrer sur celui d'un autre village ${ }^{38}$. Ce qui ressort en examinant les localisations des parcelles est une double situation des terres dans l'espace. Chaque terre est située dans un lieu-dit, lui-même inscrit dans un territoire ${ }^{39}$. La seigneurie apparaît alors découpée par une géographie du quotidien, faite d'un maillage à deux échelles. Le lieu-dit est d'essence purement locale. Il désigne une portion du territoire, pas toujours bien délimitée, de taille et d'importance variables. Il sert à se situer dans l'espace. Le territoire englobe plusieurs lieux-dits, dont l'addition forme le territoire. Chaque lieu-dit appartient précisément au territoire d'un village : cette notion est donc extrêmement précise. Chaque village se définit ainsi par un territoire qui lui est propre, qu'il entend posséder, gérer de sa propre autorité. Le village, c'est d'abord un territoire.

La résidence

21 Le territoire définit le village ; mais ce qui le distingue du simple lieu-dit est l'habitat permanent d'un groupe d'hommes. Un village se définit donc aussi par ses habitants : on est de tel village ou de tel autre. Cette notion occupe une place prédominante dans la structuration de la communauté villageoise. La manière la plus fréquente de parler des hommes d'un village, de désigner la communauté d'une localité donnée, c'est la résidence; d'où l'emploi fréquent des expressions qui disent cette résidence. Si l'on parle parfois "des hommes de tel village", on emploie de plus en plus souvent 
l'expression "les habitants de tel village ». Le conflit déjà cité de 1391 n'oppose ainsi pas deux communautés, mais les habitants des Bouchoux aux habitants de Choux et de Coirières $^{40}$. Cette notion de résidence est primordiale : c'est autour d'elle que s'opère la structuration de la communauté et du village, autour de deux réalités distinctes.

Sont habitants les gens qui demeurent au village. Cette définition simple est rappelée à l'occasion. Ainsi les moulins de Septmoncel en 1364 sont concédés aux habitatores et incolae homines nostrorum dicte parrochie Septem Moncellis, expression qui est développée plus loin dans le texte :

omnibus et singulis universis habitatoribus et incolis hominibus nostris cujuscumque conditionis aut sexus existantibus predicte parrochie nostra de Septem Moncellis qui nunc sunt vel qui pro tempore fuerint et suis heredibus et successoribus quibuscumque in prepetuum tam presentibus quam futuris [...]

Les représentants de la communauté agissant eux :

nomine suo et omnium aliorum habitatorum et incolarum dicte parrochie qui nunc sunt vel qui pro tempore fuerint et suorum heredum et successorum et posteritatis quorumcumque in perpetuum ${ }^{41}$.

Avec une telle définition, on ne risque pas d'oublier qui que ce soit! Plus simplement, les habitants sont parfois aussi dits paroissiens du même lieu : en 1467, l'abbé s'adresse aux «habitants et parrochiens de Sepmoncel et Montepyle " paroissiens du Grandvaux ", en 1476 ; cette appellation faisant suite dans le texte aux "ville, communauté et paroisse du Grandvaux »".

Cette notion d'habitants est prédominante: alors que l'on ne parle que quatre fois de communitas, le terme d'habitants est d'un emploi très fréquent, jusqu'à devenir systématique au $\mathrm{XV}^{\mathrm{e}}$ siècle. Elle n'est pourtant pas une clé d'explication de la constitution et de la composition de la communauté villageoise. La communauté et le village sont deux choses distinctes, qui doivent être examinées séparément. Les rares textes qui nous éclairent sur la communauté et sa composition font intervenir un autre critère, celui de la possession de biens. Font partie de la communauté ceux qui possèdent des biens dans le territoire du village. À ce titre, ils sont amenés à participer aux charges de la communauté, en particulier fiscales. C'est ce qui ressort de l'examen de deux textes sensiblement contemporains. En 1494, un conflit opposant les « manants et habitants de Ranchette » d'une part et différents particuliers de Chevry de l'autre, est tranché par la cour de la Cellererie de l'abbaye ${ }^{44}$. Le monastère perçoit annuellement un cens de trente pintes de miel, levé sur les « tenens terres et possessions audit lieu de Renchette pour contribuables [...] de trente pintes de miel », proportionnellement aux biens possédés. Les habitants de Ranchette contestent la répartition du cens qui a été faite, car elle ne tient pas compte des particuliers de Chevry, qui tiennent des terres à Ranchette. Une nouvelle procédure d'égalation est donc prévue, qui rassemblera « tous ceulx et celles tant généralement que particulièrement qui fréquentent des meix, maisons, terres et possessions ou finaige territoire et ville de Renchette», pour déclarer la valeur de leurs possessions, de manière à «imposer leur part dudit miel à l'équipollent de leurs dictes terres ». La redevance pèse sur le territoire d'un village ; sont invités à y participer tous ceux qui y possèdent des terres, même s'ils n'y résident pas. Au titre de la possession, ils font bien partie de la communauté et sont redevables de leur part fiscale. Les reconnaissances du village de Chaux Neuve ne disent pas autre chose en $1499^{45}$. Lors d'une reconnaissance générale des droits seigneuriaux, les 
prud'hommes, échevins et autres habitants de la Chaux Neuve contestent le montant des tailles, fixées à 25 livres estevenants, et refusent de le reconnaître, expliquant :

[que] «plusieurs particuliers de Foncine et autres tenoient et tiennent en leur territoire plusieurs pièces de terre qui vouloient être et étoient chargés de ladicte taille pour la portion qu'ils pouvoient valoir, dont nosdits seigneurs et leurs prédécesseurs avoient donné lectres à plusieurs dudit Foncine de les pouvoir tenir, leurs charges portables les uns et les autres parmy paysans, aucune charge audit seigneur; et par ainsi ne devoient-ils pas entièrement reconnoitre lesdictes 25 livres mais leur devoient estre déduit et défalqué dicelle somme ce que pouvoit monter le droit des héritages que tenoient lesdits de Foncine et étrangers auxdit territoire ".

La cause est claire: les tailles sont réparties sur la terre, et ceux de Chaux-Neuve, échevins en tête, entendent bien faire payer tous ceux qui possèdent des biens dans les limites de leur territoire, quand bien même ne résideraient-ils pas dans le village. Cette possession de biens est une notion essentielle : elle induit l'obligation de participer aux charges fiscales de la communauté, au pro rata de leurs biens possédés ${ }^{46}$. La communauté d'habitants est donc une réalité vivante, qui se définit par un territoire ; posséder des biens à l'intérieur d'un territoire entraîne une participation à ses charges, sans que l'on sache précisément si cette possession entraîne en retour l'accès aux communaux ou aux forêts, ou même la participation aux assemblées communautaires. Cette possession n'implique cependant pas l'intégration au village : ceux du village de Foncine, voisin de Chaux-Neuve, sont et restent des étrangers au village. Ils sont de Foncine, et non de Chaux-Neuve, comme ceux de Chevry ne sont pas de Ranchette. Village et communauté sont donc deux réalités distinctes: le village, régi par un principe de résidence, se distingue de la communauté, définie par la possession et l'exploitation de terres". Si par la possession de biens l'étranger semble faire partie de la communauté, et est donc à ce titre sommé de participer aux charges, il reste un étranger au village : il est de tel village, et non pas de celui-ci. On peut donc appartenir à un village, et participer aux frais d'un autre.

Se dessine alors un paysage géographique et social propre aux individus, défini justement par cette notion de village. La structure de base de la sociabilité, telle qu'elle est conçue par les paysans, n'est pas la communauté, mais bien le village ; à ce titre, si un étranger peut être admis dans la communauté, il reste toujours un étranger, ressortissant et membre d'un autre village. La communauté n'est qu'une structure de gestion des terres du territoire. C'est elle qui notamment répartit les participations aux charges fiscales de chacun, par exemple proportionnellement aux biens possédés à l'intérieur du territoire. En revanche, ce qui identifie l'individu dans la société, c'est son village, le lieu où il réside ${ }^{48}$.

28 C'est peut-être là que se situe l'explication de la relative faiblesse des structures communautaires : celles-ci ne sont finalement qu'instruments de gestion, soit pour la répartition de l'impôt, soit pour la gestion de biens communs. La véritable cellule d'identification, celle avec qui l'individu se sent en communauté, c'est le village dont il est issu, où il réside et dont il est membre. Et ici, villages et communautés ne se recoupent pas toujours; la première des communautés, plus que l'organisation municipale, c'est le village.

L'individu et la communauté : la localisation

29 Cette importance du village se marque par la définition de l'identité des individus telle qu'on peut la lire dans les textes. Sont toujours spécifiés le prénom, le nom, souvent le 
nom du père, et toujours le nom du village : l'individu est toujours attaché à un lieu géographique précis, à un territoire, distinct parfois du lieu d'habitation. Le positionnement et la dénomination de l'individu se font par rapport à cette localisation, à ce territoire précis. Le village est un identifiant très fort ; c'est celui que les hommes emploient spontanément pour se situer, à la fois géographiquement et socialement. Chaque individu se dit toujours «de tel village». Cette notion est tellement forte, qu'un individu peut aller s'établir ailleurs sans perdre cette référence : il reste quand même "de tel village». On peut le constater dans différents affranchissements: plusieurs affranchis $d u \mathrm{XV}^{\mathrm{e}}$ siècle sont dits "de tels villages, résidant à Saint-Claude "; on précise donc le village d'origine, l'affranchissement venant ici sanctionner un état de fait, le changement de résidence. Mais on rencontre aussi à diverses reprises des individus dits de tel village, et résidant dans un autre. C'est le cas par exemple de Jean Monnier, de Marignat, résidant à Molinges ${ }^{49}$, ou de Claude Michalet, de Choux, habitant Chaumont ${ }^{50}$. Ici, c'est bien le village d'origine qui est rappelé, même si les liens entre l'individu concerné et son village d'origine ont pu se distendre. Le lieu d'habitation et le territoire d'origine sont deux réalités distinctes, qui tous deux servent à identifier l'individu. Mais dans l'ordre de présentation, c'est le territoire d'origine qui est mis en avant.

Villages et communautés sont deux notions différentes, et présentées comme telles. Une communauté peut par exemple représenter un seul village, comme à Lavancia, ou plusieurs : c'est le cas de celle de Longchaumois et Orsière, qui élisent en commun des " sindics ", mais qui constituent bien deux villages distincts ; idem pour Septmoncel et Montépile. Un autre exemple est offert par les « échevins, prudhommes et conseillers des ville, communauté et paroisse du Grandvaux ». Il s'agit ici d'une zone géographique et orographique distincte, représentée par un seul organisme, même si différents villages peuvent être dénombrés sur ce plateau: l'Abbaye, Fort-du-Plasne, La Chaumusse $^{51}$ par exemple.

Cette notion de village, des plus difficiles à définir, est donc essentielle pour l'individu et son positionnement géographique et social. Mais ce territoire auquel se réfère l'individu n'a pas toujours la même réalité d'une zone à l'autre de la Terre de SaintClaude. Son échelle peut varier considérablement, sans qu'une quelconque constante puisse être mise en évidence. Il semble bien qu'ici la géographie à laquelle se réfèrent les individus soit une création de ses habitants plus qu'une réalité administrative quelconque. Tous les cas semblent possibles : s'il arrive que le village s'identifie à la paroisse, ce n'est pas la règle. On a vu aussi que parfois la communauté, regroupant plusieurs villages, s'identifiait alors à la paroisse: c'est le cas en Grandvaux, à Longchaumois, à Septmoncel. La solidarité paroissiale n'est pas un fait acquis : Chevry et Ranchette, en conflit au sujet d'un cens de miel, font partie de la même paroisse, Saint-George, à laquelle participent aussi une dizaine d'autres villages.

Rares sont finalement les paroisses qui correspondent à un village donné ; le territoire de la paroisse ne s'identifie pas au finage du village ${ }^{52}$. Le village est en général une unité de taille inférieure à la paroisse. Il ne correspond pas à une structure administrative seigneuriale : celle-ci a plutôt tendance à s'identifier au réseau paroissial. C'est le cas en particulier en montagne, où les divisions paroissiales recoupent les divisions seigneuriales : le Grandvaux, Les Bouchoux, La Mouille, Mouthe sont tout à la fois des seigneuries où réside un prieur, des paroisses de la Terre de Saint-Claude, et le siège d'une prévôté, couvrant parfois des étendues considérables, où les habitants sont 
regroupés en villages lâches, distants les uns des autres de parfois plusieurs heures de marche. La communauté villageoise, telle qu'elle apparaît dans les rares textes où elle est mentionnée, semble finalement se calquer plus sur les structures seigneuriales et paroissiales que sur les structures d'habitat, de peuplement ou d'exploitation des terres.

33 La notion de village dessine une géographie qui est particulière, ni administrative ni physique, mais qui est le résultat du cadre de vie des paysans tels qu'ils l'ont façonné. C'est le réseau des villages qui constitue la réalité vécue du réseau communautaire. Les individus expriment leur appartenance à un ensemble précis, formant la cellule de base de la géographie sociale de la Terre de Saint-Claude. Cette géographie est bien celle des paysans, et non celle du seigneur : elle ne correspond ni au le réseau administratif seigneurial, ni au le réseau paroissial.

L'insertion dans la seigneurie

34 Ce réseau des villages s'intègre dans un maillage plus large : celui de la paroisse et celui de la seigneurie locale, prévôté ou prieuré. Cette première dimension est éclipsée par une dimension plus importante. Au-delà du cadre administratif, ce réseau s'intègre aussi dans une forme supérieure de communauté, celle de la seigneurie, formée ici par la Terre de Saint-Claude: on est dans la Terre ou on ne l'est pas. Signe de cette appartenance : c'est d'abord vers l'abbé que l'on se tourne en cas de conflit. Le réflexe est logique dans le cadre interne de la seigneurie san-claudienne: quand deux communautés ou deux villages de l'abbé s'opposent, on fait appel à la justice seigneuriale pour départager les plaignants, les représentants de chaque communauté défendant leur cause.

35 Il est un cas cependant où les communautés ne se font pas représenter par leurs échevins, mais directement par l'abbé. Lors de conflits avec les gens de Gex et de $\mathrm{Nyon}^{54}$, dans la plaine suisse, dont les avancées dans les forêts jurassiennes au cours du $\mathrm{XIV}^{\mathrm{e}}$ siècle les amènent à rencontrer les paysans des communautés de Longchaumois, des Bouchoux ou de Septmoncel, c'est vers l'abbé que ces communautés se tournent. Au lieu de désigner des représentants de la communauté, c'est l'abbé qui devient lui-même représentant de ces communautés face à l'extérieur. C'est vers lui qu'on se tourne, et c'est de lui qu'on attend la solution. Ce sont donc des représentants du seigneur qui vont négocier avec les seigneurs ou la ville de Gex un accord de limitation des pénétrations dans les forêts jurassiennes.

L'individu et la famille

36 Il est rare qu'un individu se définisse par son seul nom dans le village. La communauté familiale, très forte dans la montagne jurassienne, est un élément incontournable dans la dénomination des personnes : l'individu se situe toujours dans un espace familial et lignager. C'est parfois le nom du père qui est précisé ; c'est le cas en 1313, où le couvent accense à Nicolas, fils de Jean Suffisat, et à Guichardette sa femme, un emplacement pour y construire un moulin $^{55}$; en 1378 encore, où l'aumônier accense un pré en Haut Crêt à Pierre, fils de feu Poncet Sapel, de Chaumont ${ }^{56}$; en 1419, où Piere fil de Pieret li Guyan passe reconnaissance de certaines redevances ${ }^{57}$. Cette précision est d'ailleurs systématique pour les femmes, où on rappelle les noms du père et du mari ${ }^{58}$.

37 Avec le nom du chef de la famille, du père, sont parfois aussi mentionnés les autres membres de la famille qui vivent sous le même toit, frères, sœurs ou cousins, solidaires des redevances dues par l'exploitation et formant la cellule de base de la vie sociale. 
Cette communauté est le premier maillon des structures qui encadrent et situent l'individu. En 1491, Étienne Maillet passe une reconnaissance de dettes auprès de l'aumônier, «tant en son nom que en celui de Pierre Maillet son frère et aussi en celui de Clauda et de Claire Maillet ses seurs, tous de Chaumont " : c'est bien la famille qui est engagée, à travers son représentant mâle. Cette unité familiale est dirigée par un chef de famille, qui agit au nom de la communauté. Les reconnaissances passées dans le Terrier de la Pitance dans les années 1503-1508 mentionnent systématiquement les membres de la communauté familiale :

«Jehan David, de Chaulmont, fil de fust Jehan David, tant en son nom comme pour et en nom de Jehannin et Claude David, ses frères, et de Pierre et Claude David, leurs cousins, personnes conjointes et commun en biens, absents, soy faisant fort deulx quant à ce, saiges sachant et de son bon gré et volonté, pour lui et ses hoirs, sesdits frères et cousins et promettant les faire consentir au contenu des présentes se mestier est, cognoit et confisse tenir pourter et possider [...] $\aleph^{59}$.

Cette communauté familiale, renforcée par la possession en indivis des biens familiaux $^{60}$, est marquée par la présence de maisons au sens anthropologique du terme, appelée dans le Jura hôtel, hostal, chasal, maison ou meix ${ }^{61}$. Les hommes qui passent reconnaissance, ceux qui forment la communauté du village, sont les maîtres de ces hostaux ${ }^{62}$. Les habitants du village sont en fait les membres des hostaux du village. En 1480, lors des reconnaissances des droits seigneuriaux à Châtelblanc, si tous les habitants sont convoqués, il est spécifié que seuls jurent «tous les chefs d'hostels de ladite ville ». En 1476, les habitants et paroissiens du Grandvaux demandent à ce que les journées de justice débutent dès le matin afin qu'ils puissent rentrer « de jour en leur hostel ». Cette notion d'hostal est d'ailleurs déjà la cellule de base de l'imposition fiscale au début du XIVe siècle. C'est encore ce même terme qu'emploie en 1487 Pierre Dronier dans son testament, pour désigner la maison et la communauté familiale de ses cousins et neveux, à qui il lègue ses droits et sa part dans l'indivision :

« item je donne et lègue [...] aux enffans masles de Claude Dronier l'ancien et jadis mon neveux, et à Claude Dronier, demeurant audit Ravillolles et à Etienne Dronier demeurant à Orgelet, frères et enffans de feu Jehan Dronier, jadis mon frère, et aussi mes biens amez nepveux, assavoir auxdits enfans dudit feu Claude et audit Claude Dronier, lesquelx sont manants et demourans et mariés audit Ravillolles en l'ostel et sur le meix de feurent mes pères et mères [...] tous et singuliers mes biens mobles, héritages, droiz, action et partage à moy advenuz par le décès et trespas de mesdits feurent père et mère paternelz et maternelz quelxconques estant par indivis en communion avec eulx par ensemble oudit hostel, territoire dillec et lieux circonvoysins, sans rien y retenir en propriété $»^{63}$.

Il s'agit ici d'une stratégie familiale développée pour éviter la mainmorte ${ }^{64}$. Ayant rompu la communion initiale, Pierre Dronier menace la survie de celle-ci. Il s'en retire donc par son testament et abandonne ses droits à ceux qui continuent de vivre dans la communion familiale initiale, de manière à éviter l'échute des biens familiaux, totale ou partielle, au seigneur. Mais à travers cette attitude, on saisit bien l'importance de la communauté familiale pour l'individu : devenu prêtre, curé de Gevingey, près de Lonsle-Saunier, Pierre Dronier n'a plus que des liens lâches avec la communauté familiale ; c'est pourtant elle qu'il privilégie, ne léguant que de faibles sommes à ses autres neveux, issus du même lignage, mais installés à Dijon ou Besançon. La référence au village et à la famille est ici un signe fort d'identification sociale.

La structuration des communautés et le positionnement de l'individu 

donnent les archives seigneuriales ne signifie pas que celles-ci soient inexistantes. Mais si les représentations communautaires existent, elles sont avant tout un instrument de gestion et de représentation des villages, assurant le lien entre le réseau seigneurial et les paysans. La communauté telle que la ressentent et la vivent les individus n'est pas la communauté administrative, municipale, telle que peuvent la révéler les conflits d'intérêts. La majorité de ces conflits tournent autour des redevances dues par le village : les étrangers à la commune sont priés de participer, au pro rata des biens possédés dans le territoire. Il y a donc un territoire communal, et c'est la possession de biens dans ce territoire qui entraîne la participation aux charges.

41 et dont les maillons lui sont parfaitement connus. Il se situe par rapport à ce réseau via une triple identification. Le premier groupe est celui de la famille, au sens large. La communauté familiale est la cellule de base de la vie sociale et du positionnement de l'individu. C'est elle qui sert de base à la représentation dans la communauté villageoise. Le second groupe auquel l'individu se réfère est le village, caractérisé par un territoire précis, et par ses habitants. C'est en effet par la résidence, comprise comme membre d'une des communautés familiales du village, que l'on appartient à un village. Le dernier élément est celui de l'insertion dans un ensemble seigneurial plus vaste, celui de la Terre de Saint-Claude. Chaque individu est homme de l'abbé, soumis à la tutelle seigneuriale, lié par la coutume dans un statut unique et contraignant.

De ces trois étages, c'est le village qui est le plus déterminant : ce seul élément suffit à situer un individu. Il est tellement prégnant qu'il perdure même si l'individu s'installe dans un autre village. Villages et communautés sont deux choses distinctes: les structures communautaires ne reflètent pas forcément les sentiments d'appartenance à une communauté. Il y a d'une part la communauté administrative, celle chargée par le seigneur de répartir les redevances; il y a d'autre part la communauté vécue et ressentie, celle du village, dont la prégnance est beaucoup plus forte. Le maillage des villages constitue un réseau créé par les individus eux-mêmes. Il s'agit bien d'un réseau du vécu plus que d'un réseau administratif ou religieux : la géographie des seigneurs et celle des paysans ne correspond pas toujours.

\section{NOTES}

1.Ou plutôt Terra Sancti Eugendi, terre de saint Oyend, en latin, nom employé pendant tout le Moyen Âge. L'appellation Terre de Saint-Claude est ultérieure, mais c'est elle qui est restée dans le vocabulaire courant. Pour des raisons de commodité, c'est l'expression que j'emploierai (avec une majuscule) pour désigner la terre soumise à la juridiction de l'abbé et qui constitue le cadre de mon étude.

2.Pour l'essentiel, les archives de l'abbaye et de la Terre de Saint-Claude sont aujourd'hui conservées aux Archives Départementales du Jura, à Lons-le-Saunier (ADJ, série $\mathrm{H}$, sous-série $2 \mathrm{H}$ : Fond de l'Abbaye de Saint-Claude). L'inventaire de ce fonds a été

Cahiers de recherches médiévales, 10 | 2003 
réalisé par Henri Hours, alors conservateur des Archives Départementales du Jura, et publié en 1994 : Répertoire numérique de la série H, sous-série $2 \mathrm{H}$ : Fond de l'Abbaye de SaintClaude, Lons-le-Saunier, 1994.

3.La charte de 1310 (ADJ 2 H 342 ; nombreuses copies sous d'autres cotes) fait référence à des franchises antérieures inconnues. Elle est ensuite complétée en 1330 et confirmée en 1393.

4.Confirmation et extension de franchise antérieures inconnues ; ADJ, 2 H 925.

5.Sur environ 80 villages recensés dans la terre de Saint-Claude au début du XIV siècle. C'est un chiffre a minima, établi à partir de divers documents : Livre d'Or (copie dans ADJ, 2 H 157) et pouillé d'Innocent IV (1245; ADJ, 2 H 9) principalement. D'autres textes éparts faisant mention de villages attestés au début du XIV ${ }^{\mathrm{e}}$ siècle ont pu compléter ponctuellement cette évaluation. Ce chiffre est sous-évalué : d'autres villages apparaissent au cours des XIV ${ }^{e}$ et $\mathrm{XV}^{\mathrm{e}}$ siècles ; surtout, de nombreuses communautés attestées regroupent plusieurs villages distincts, comme dans le Grandvaux. Mais il est impossible de savoir à quel moment de simples écarts deviennent de vrais villages : les habitants de cette zone se désignant par exemple toujours comme originaires « du Grandvaux ", sans plus de précision, il est difficile de savoir à une époque donnée combien de villages contient cette région.

6.Longchaumois (ADJ, $2 \mathrm{H}$ 732).

7. « Nos [...] concessimus [...] omnes ipsi aut saltem maior pars habitantium dictarum villarum et eorum heredes possint eligere de tribus annis in tribus annis quatuor probos viros seu sindicos qui negocia tractare et supportare possint et valeant, ac taillias modo antiquo et caetera subsidia jactare prout eis videbitur expedire absque tamen diminutione dictarum terrarum temporis moderni nisi dumtaxat de concensu nostro » (ADJ, $2 \mathrm{H} 732$ ).

8.Les mêmes communautés de Longchaumois et Orsières avaient bénéficié en 1301 d'un abonnement général des redevances, contre le versement d'une indemnité de 300 livres viennoises et une rente annuelle et perpétuelle de 60 livres viennoises (ADJ, 2 H 706).

Mais à aucun moment dans ce texte il n'est fait référence à une quelconque forme d'organisation municipale : on ne sait ni comment ni par qui sera répartie et levée cette redevance annuelle, preuve qu'à cette époque le fonctionnement des institutions municipales est encore inexistant. Il faut attendre la charte de 1393 pour le voir apparaître.

9.Odile Redon note au contraire que le terme de commune est systématiquement employé pour désigner un village et ses habitants dans les traités avec leur seigneur dans la région siennoise au XIII ${ }^{\mathrm{e}}$ siècle (O. Redon, « Seigneurs et communautés rurales dans le contado de Sienne au XIII siècle », MEFREM, 91, 1979, 1 : p. 149-196, 2 :

p. 619-657.

10.Franchises de Mouthe, 1296 (ADD, 35 H 4) ; Châtelblanc, affranchi en 1303 par Jean de Chalon et en 1351 par l'abbé (ADJ, 2 H 160) et Rochejean en 1364 (2 H 160).

11.Actes d'association conclus entre Jean de Chalon et l'abbé de Saint-Claude (ADJ, $2 \mathrm{H}$ 1255).

12.Les limites des terres communes sont précisées dès la charte de 1310, mais il faut attendre la mise au point de 1330 pour que tous les points litigieux soient tirés au clair. Les limites des communaux laissés à la libre disposition des bourgeois sont alors reprécisées, ainsi que les dates de mises en défens. La communauté a donc explicitement la libre gestion des terres communes. (ADJ, 2 H 342 ; copie de ces titres en 1 Mi 1009, ZZ 38 et ZZ 39). 
13.Exemples aux Bouchoux en 1391 (ADJ, 2 H 833), en 1412 à Saint-Lupicin (ADJ, $2 \mathrm{H}$ 158), Lavans, 1414 (ADD, 7 H 2), à Septmoncel et Longchaumois en 1453 (ADJ, 2 H 688), à Septmoncel en 1467 (ADJ, 2 H 158).

14.Dans les reconnaissances du Terrier de la Pitance, seigneurie du réfecturier de l'abbaye (aujourd'hui conservé aux Archives municipales de Saint-Claude ; non-coté), les confronts des parcelles laissent apparaître de temps à autre la présence de communs, sans que cela soit une règle. Sur les 33 villages où des parcelles sont délimitées, seuls 11 mentionnent des communaux dans les confronts.

15.La communauté des habitants réclame le droit de mener paître son bétail sur les terres vacantes et non cultivées du village. Les officiers contestent ce droit en vertu du droit de mainmorte du seigneur : toute terre à la mort de son tenancier fait l'objet d'échute au seigneur. La communauté se fait finalement reconnaître ce droit de vaine pâture, ainsi que la libre disposition des terres vacantes : l'abbé concède « quod ipsi et eorum heredes deinceps et in perpetuum eorum vana pastura communi per eorum animalium utantur et gaudeant in omnibus et singulis terris et locis vacantibus et existentibus in dimeria nostra de Lavan [...] et quod in omnibus locis in quibus bono modo et utiliter bladum crescere non potest in eorum dimeria de Lavvan ipsi homines nostri de dicta dimeria fenum facere possint et eorum heredes perpetuo » (ADD, $7 \mathrm{H} \mathrm{2}$ ).

16.À plusieurs reprises, l'abbé se plaint de la stérilité des terres, de leur faible rapport, de la difficulté à les mettre en culture, et du fait que ces terres sont inutiles tant qu'elles ne sont pas cultivées. C'est ce qui ressort des préambules des chartes d'associations de 1266, 1299 et 1301 avec Jean de Chalon et Étienne de Thoire-Villars (ADJ, 2 H 1109 et 2 H 1255), et des concessions accordées à Longchaumois en 1390. 17.ADJ, 2 H 998.

18.ADJ, 2 H 1032.

19. Habitatores villarum de Choux, Parrochie Sancti Georgii, et de Curières, tam in pascuis communibus ipsorum conquerentium, quibus ipsi conquerentes per se et eorum predecessoribus usi fuerunt in eorum territorio et territoriis dictarum villarum de Choux, parrochie Sancti Georgii, et de Curières, quam etiam in viis publicis itineribus et sentieriis tendentibus per territorio ipsarum villarum ipsos conquerentes perturbant, molestant et inquietant, taliter quod bono modo ipsi conquerentes de ipsis pascuis usi et gaudere non possunt [...] nec eorum negocia et necessitates per dictas vias publicas itinera et senteria facere possunt, quod cedit et redundat in grande prejudicium damnumque non modicum (ADJ, $2 \mathrm{H} \mathrm{870).}$

20.On peut d'ailleurs remarquer à ce sujet que le milieu du XIV siècle ne représente sur ce point en cas une coupure marquante. Comme le remarquait Jean-Paul Boyer dans la vallée de la Vésubie, les conflits entre communautés ne sauraient se résumer à une question de pression démographique, puis réglés à partir du milieu du siècle par une crise dont on ne distingue que peu de traces dans la documentation san-claudienne (JP. Boyer, Hommes et communautés du haut pays niçois médiéval : la Vésubie (XIII ${ }^{e}$ $X V^{e}$ siècle), Nice, 1990).

21.ADJ, 2 H 158, fol. 241 (Saint-Lupicin), et ADD, 7 H 2 (Lavans).

22.ADJ, 2 H 521.

23.ADJ, 2 H 158, fol. 211.

24. "Gentilshommes et prodommes des dictes villes » [de Coiron et Meussia], 1365 (ADJ, $2 \mathrm{H}$ 998).

25. À Chaumont, en 1505 (ADJ, ZZ 37).

26. À Septmoncel en 1467, à Mouthe, en 1488 (ADD, 35 H 5 ; représentants attestés depuis 1296, ADD, 35 H 4), à Chaux-Neuve en 1499 (ADJ, 2 H 160, fol. 31). 
27.Dans le Grandvaux, en 1476 : prud'hommes, échevins et conseillers des ville, communauté et paroisse de Grandvaux (ADJ, $2 \mathrm{H} \mathrm{160,} \mathrm{fol.} \mathrm{59).}$

28.Attesté en 1258 (ADJ, 2 H 894), 1347 (ADJ, 2 H 521), 11385 (ADJ, 2 H 989) et 1448 (ADJ,

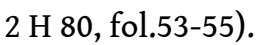

29. Attesté en 1244 (ADJ, 2 H 962), 1250 (id.), 1266 (ADJ, 2 H 963), 1267 (ADJ, 2 H 940), 1304 (ADJ, 2 H 911), 1317 (ADJ, 2 H 940), 1438 (ADJ, 2 H 315).

30.On retrouve le terme de finage, employé seul (sou la forme finagium villae) en 1205 (ADJ, 2 H 963), 1237 (ADJ, 2 H 1016), 1244 (ADJ, 2 H 962, 1260 (ADJ, 2 H 514), 1264 (ADJ, 2 H 658), 1297 (ADJ, 2 H 894). Le dernière mention date de 1308 (ADJ, 2 H 49). Par la suite, il est systématiquement employé en binôme avec territoire, sous la forme finagium seu territorium.

31.Seuls quatre villages, en-dehors de la ville de Saint-Claude, sont qualifiés de communitas : Lavancia en 1347 et 1397 (ADJ, 2 H 520 et 2 H 521), Lavans en 1414 (ADD, 7 H 2), le Grandvaux en 1476 (ADJ, 2 H 160, fol.59), Septmoncel en 1478 (ADJ, 2 H 808). 32.En 1364, dans l'acte déjà cité concernant les moulins de Septmoncel. Les représentants de la communauté sont dits procuratores electi et probati nominati per universitatis dicte parrochie (ADJ, 2 H 817). Même la ville de Saint-Claude, dont les bourgeois forment la communauté la plus organisée face au pouvoir de l'abbé, n'est jamais appelée universitas.

33.Expression que l'on retrouve dès 1258 (ADJ, $2 \mathrm{H} 1024)$, mais qui est surtout courante au XIV ${ }^{\mathrm{e}}$ siècle. On la retrouve en 1303 (ADJ, 2 H 929), 1310 (ADJ, 2 H 546), 1351 (ADJ, 2 H 923), 1371 (ADJ, 2 H 991).

34. ADJ, 2 H 936.

35. ADJ, 1 Mi 1008, ZZ 38.

36.ADJ, 2 H 1035, fol. 23.

37.Georges Sivéry définit le finage comme la totalité de la superficie d'un village, le terroir comme la seule partie cultivée ou mise en valeur de ce finage (G. Sivéry, Terroirs et communautés rurales dans l'Europe occidentale au Moyen Âge, Lille, 1990, p. 33). Cette distinction ne peut s'appliquer au Moyen Âge : les expressions finagium seu territorium, finagium sive territorium ne laissent guère de doute quant à l'équivalence des deux notions.

38.Pierre de Saint-Jacob soulignait déjà cette adéquation entre village, finage et territoire en Bourgogne (P. de Saint-Jacob, Les paysans de la Bourgogne du nord au dernier siècle de l'Ancien Régime, Paris, 1960, rééd. Rennes/Dijon, 1995, p. 76), et insistait sur cette communauté territoriale : « le groupe se détermine largement par l'espace qu'il occupe et la circonscription qu'il contrôle » (op. cit., p. 75).

39.Exemple en 1405, dans un échange entre particuliers de terre à Chaumont : unum curtilem suum situm in territorio Calvomontis in loco dicto en Leschalier (ADJ, $2 \mathrm{H} \mathrm{396).} \mathrm{Ce}$ système de localisation se fait systématique au $\mathrm{XV}^{\mathrm{e}}$ siècle ; il est utilisé pour situer toutes les parcelles dans le Terrier de la Pitance, au début du XVI ${ }^{\mathrm{e}}$ siècle.

40. Habitantes ville dicti loci de Boschoux d'une part, et habitatores villarum de Choux et de Curières de l'autre (ADJ, 2 H 870).

41.ADJ, 2 H 817.

42.ADJ, 2 H 158, fol. 211.

43.ADJ, 2 H 160, fol. 59.

44.ADJ, $2 \mathrm{H} 484$.

45.ADJ, 2 H 160, fol. 31.

46.C'est la procédure déjà signalée pour Ranchette d'esgalacion, ou d'egalement. 
47. Odile Redon note au contraire que l'appartenance à la commune obéit à ce principe de résidence ; mais dans le Siennois, village et commune n'offrent pas de différences majeures (op. cit.). Il semble que le schéma jurassien soit aussi sensiblement différent de ce que Pierre de Saint-Jacob constatait en Bourgogne : pour lui, l'adéquation entre résidence et exploitation est constitutive de la communauté (op. cit., p. 76). La dissociation que j'observe dans le Jura doit encore être expliquée ; le fait que l'on se situe dans des terres de conquête, peu densément peuplées, est peut-être un premier facteur explicatif.

48. Cette importance de la notion de résidence dans la constitution d'un sentiment d'appartenance à une même communauté est soulignée en Franche-Comté par Bernard Derouet : «Territoire et parenté. Pour une mise en perspective de la communauté rurale et des formes de reproduction familiales ", Annales HSS, Mai-Juin 1995, n 3, pp. 645-686.

49.En 1459 (AMSC, BB3, fol. 381).

50.En 1476 (AMSC, BB1, fol. 199).

51.Dès 1385 pour Fort-du Plasne (ADJ, 2 H 989), 1440 pour la Chaumusse (AMSC, BB1, fol. $3 \mathrm{v}$ ). Il est possible toutefois qu'il ne s'agisse encore que de hameaux très lâches à cette époque, ce qui expliquerait alors cette représentation commune.

52.Colette Merlin soulignait aussi cette inadéquation entre les territoires du village et de la paroisse, et le sentiment très fort d'appartenance au village (C. Merlin, Ceux des villages. La société rurale dans la « Petite Montagne " jurassienne à la veille de la Révolution, Cahier d'Études Comtoises, 52, et Annales Littéraires de l'Université de Besançon, 523, Besançon, 1994).

53.Odile Redon note elle une adéquation parfaite entre la commune, le district seigneurial et l'espace vital de la communauté (op. cit.).

54. Nombreux conflits au cours du XIV ${ }^{\mathrm{e}}$ siècle : l'abbé traite avec l'abbaye de Chézery en 1308,1315 et 1322 ; avec les seigneurs de Gex en 1334, avec la ville de Gex en 1389 (ADJ, $1 \mathrm{Mi}$ 1009, ZZ 38).

55.ADJ, 2 H 490.

56.ADJ, 1Mi 1009, ZZ37.

57.ADJ, 2 H 274.

58.Selon la forme Perrinette fille de feut Estienne Magnin femme dudit Pierre Crestin (en 1421, ADJ, 1Mi 1009, ZZ 37). On retrouve ici les mêmes caractéristiques que Jacques Heers mentionnait déjà dans les familles de l'élite génoise vers 1400 : la mention du nom du père se généralise, en complément du nom de l'individu. Il remarque à ce titre que « le fait de marquer la filiation est un signe social nettement affirmé » (p. 61). Il mentionnait aussi la présence systématique des noms du mari et du père pour les femmes, même veuves ; autant de marques d'appartenance sociale à un clan familial défini que l'on retrouve dans la société rurale jurassienne à partir du XV $\mathrm{XV}^{\mathrm{e}}$ siècle. (J. Heers, La clan familial au Moyen Âge. Études sur les structures politiques et sociales des milieux urbains, Paris, 1974. Le poids des structures familiales a aussi été mis en évidence par Pierre Dubuis dans le Valais suisse : Les vifs, les morts et le temps qui court. Familles valaisannes, 1400-1550 (Cahiers lausannois d'histoire médiévale, 16), Lausanne, 1995.

59.Reconnaissance passée en 1504 (AMSC, Terrier de la Pitance, fol. 31).

60.Selon un système proche de celui que l'on retrouve dans le Faucigny, terre elle aussi soumise à une mainmorte générale au Moyen age ; voir N. Carrier, La vie montagnarde en Faucigny à la fin du Moyen Âge, Paris, 2001, chap. V. Sur ces communautés familiales, voir aussi J. Gaudemet, Les communautés familiales, Paris, 1963. 
61.On retrouve l'importance de la maison, signalée aussi par Jacques Heers, en liaison avec le nom du clan qui la tient (op. cit., p. 67). Les parentés avec les réalités pyrénéennes, tel qu'elles peuvent apparaître à Montaillou, sont évidentes (E. LeroyLadurie, Montaillou, village occitan, Paris, 1975). Il est à noter d'ailleurs que le meix garde son nom un certain temps, même après abandon et démembrement au profit de la communauté ; plusieurs exemple dans le Terrier de la Pitance, dont le Meix Gravet, à Chanon, partagé entre plusieurs propriétaires suite à son démémbrement à une date inconnue (AMSC, non-coté). Ce même phénomène est remarqué par J-C. Hélas dans le Gévaudan; il souligne qu'on ne parle jamais « d'un manse », mais du « manse de Untel » (J-C. Hélas, « Le manse en Gévaudan au milieu du XV $\mathrm{XV}^{\mathrm{e}}$ siècle », Cadres de vie et société dans le Midi médiéval, Hommage à Charles Higounet, Annales du Midi, 1990, pp. 173-179).

62.Au contraire de ce que Odile Redon remarque dans le Siennois, où l'assemblée générale des habitants de la commune regroupe tous les hommes majeurs, et pas seulement les chefs de famille (op. cit.).

63.Testament de l'officialité de Besançon, publié par U. Robert, Testaments de l'officialité de Besançon, 1265-1500. T. 1 : 1265-1400, Paris, 1902, T. 2 : 1400-1498, Paris, 1907, vol. 2, p. 123.

64.La coutume san-claudienne stipule en effet un statut servile généralisé pour tous les tenanciers du monastère, avec échute des biens en cas de mort sans descendant direct maintenant la communauté familiale en ses murs. Sur ces questions d'héritage et de stratégies familiales en Franche-Comté, voir de Bernard Derouet, en plus de l'article déjà cité : « Pratiques successorales et rapports à la terre : les sociétés paysannes d'Ancien Régime », Annales ESC, 1989, pp. 173-206 ; « Le partage des frères. Héritage masculin et reproduction sociale en Franche-Comté aux XVIII ${ }^{\mathrm{e}}$ et XIX ${ }^{\mathrm{e}}$ siècles ", Annales ESC, 48, 2 (mars-avril), 1993, pp. 453-474 ; « Transmettre la terre. Origines et inflexions récentes d'une problématique de la différence ", Histoire et Sociétés rurales, $\mathrm{n}^{\circ} 2,2^{\mathrm{e}}$ semestre, 1994, pp. 33-67. Voir aussi l'ouvrage de Michèle Dion-Salitot, Héritage, parenté et propriété en Franche-Comté du XIII ${ }^{e}$ siècle à nos jours, Paris, 1988.

65.Liste des abréviations :

ADJ : Archives départementales du Jura.

ADD : Archives départementales du Doubs.

AMSC : Archives municipales de Saint-Claude.

\section{AUTEUR \\ VINCENT CORRIOL}

Université Paris-I 